\title{
A framework and tool for supply network strategy operationalisation
}

\begin{abstract}
This research aims to develop a framework to capture the interplay of network strategic decisions, and to understand how firms operating within a supply network interact with each other. The research involves an in-depth longitudinal case study in one supply network consisting of 20 firms. Data was systematically collected through the use of a Supply Network Analysis Process (SNAP) methodology. Interviews and secondary data were collected for further data validation and triangulation. This paper describes the above case study in detail, explains the SNAP methodology and framework development, and discusses the implications of the research both in academia and industry.
\end{abstract}

Keyword: Supply network, SNAP, Strategy interplay 